\title{
STATUS of Nb-Pb SUPERCONDUCTING RF-GUN CAVITIES
}

\author{
J. Sekutowicz, J. Iversen, D. Klinke, D. Kostin, W. Möller, A. Muhs, DESY, Hamburg, Germany \\ P. Kneisel, TJNAF, Newport News, USA \\ J. Smedley, T. Rao, BNL, Upton, USA \\ P. Strzyżewski, A. Soltan INS, Swierk/Otwock, Poland \\ Z. Li, K. Ko, L. Xiao, SLAC, Menlo Park, USA \\ R. Lefferts, A. Lipski, SUNY, Stony Brook, USA \\ M. Ferrario, INFN, Frascati, Italy
}

\begin{abstract}
We report on the progress and status of an electron RFgun ${ }^{*}$ made of two superconductors: niobium and lead [1]. The presented design combines the advantages of the RF performance of bulk niobium superconducting cavities and the reasonably high quantum efficiency of lead. The design of RF-gun and performance of 3 test cavities without and with the emitting lead spot are reported in this contribution. Measured quantum efficiency for lead at $2 \mathrm{~K}$ is presented briefly. More details are reported in [9].
\end{abstract}

\section{INTRODUCTION}

\section{Motivation}

Improvement in RF performance of superconducting (sc) cavities over the past decade has made feasible continuous wave (cw) and near-cw operations of superconducting electron linacs at high accelerating gradients. Both operation modes require injectors operating at $\mathrm{cw}$ or near-cw, providing low emittance electron beams. An example of such an injector (so called split injector) is discussed in [2]. The most demanding component of a cw injector is the RF-gun, operating in a cw mode and delivering highly populated $(\sim 1 \mathrm{nC})$ low emittance bunches. When generating highly populated low emittance bunches, both room temperature and superconducting RF-guns have to be operated at high accelerating gradients to suppress space charge effects that dilute the emittance. Normal conducting RF-guns face difficulties in meeting this requirement in the $\mathrm{cw}$ or near-cw mode. Their copper walls dissipate many kilowatts of power in fulfilling high gradient conditions even when they operate at low pulse repetition rate. Superconducting RF-guns (SRF-guns) dissipate orders of magnitude less power than the normal conducting devices. They can be operated at high duty factor. The challenge here is integration of a non superconducting photo-cathode into a sc cavity [3] in a way that preserves its original high intrinsic quality factor $Q_{o}$ (small cryogenic losses). One possible solution to this problem, based on a choke filter design, is investigated at Forschungszentrum Rossendorf [4, 5]. Another approach, very attractive and technically feasible for miliampereclass SRF-guns, is to use a superconducting material as

\footnotetext{
This work has been partially supported by the EU Commission, contract no. 011935 EUROFEL-DS5 and US DOE under contract number DE-AC02-98CH10886.
}

the photo-cathode. In this case, difficulties arise from the moderate quantum efficiency $(Q E)$ of the superconducting materials, which must be compensated with shorter wavelength and higher pulse energy of the illuminating laser. The niobium cathode proposed and tested at BNL [6] demonstrates rather poor $Q E$. A complementary approach with lead [7] is discussed in the following section.

\section{LEAD QE AND RF-DESIGN}

\section{QE of lead}

Lead has superior $Q E$ to niobium. Test results of lead $Q E$ at room temperature were reported by us in [8]. Fig. 1 shows the summary of the tests at $300 \mathrm{~K}$ vs. photon energy $E_{p}$ for niobium, bulk lead and lead samples deposited with various techniques. The highest $Q E$ of $0.55 \%$ has been measured for the arc-deposited sample illuminated with $6.5 \mathrm{eV}$ photons. For $5.8 \mathrm{eV}$ photons $\left(5^{\text {th }}\right.$ harmonics of $1064 \mathrm{~nm}$ infrared laser) the $Q E$ of that sample was still $0.25 \%$. $Q E$ of the electroplated and magnetron deposited samples at this photon energy is $\sim 0.17 \%$. Generation of $1 \mathrm{nC}$ bunch with this $Q E$ will require $3.4 \mu \mathrm{J}$ energy per pulse on the cathode. The cold $Q E$ test was done very recently and is reported elsewhere in these proceedings [9].

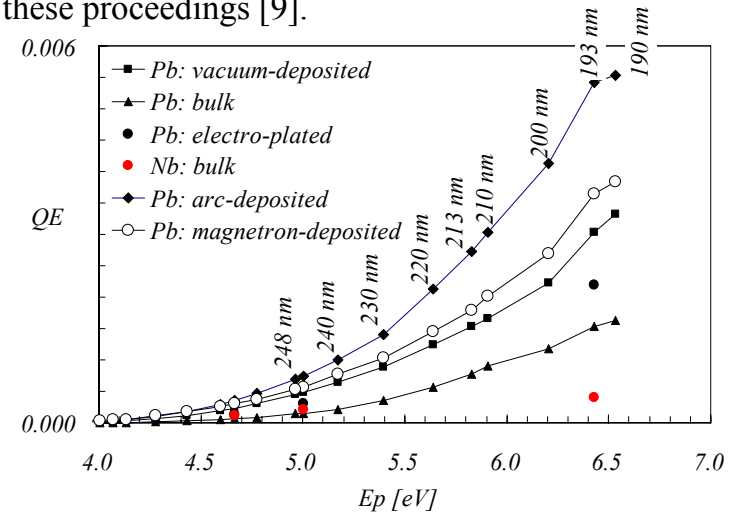

Figure 1: Measured $Q E$ of lead deposited with various coating methods. Bulk $\mathrm{Pb}$ and $\mathrm{Nb}$ data are displayed for comparison.

\section{$R F$-design}

In the hybrid $\mathrm{Pb}-\mathrm{Nb}$ gun, a small emitting spot of lead $(\varnothing<3 \mathrm{~mm})$ will be located in the center of the back wall of the 0.6 -cell of a 1.6-cell ${ }^{\dagger}$ cavity (Fig. 2), which will be

† The first cell is $0.6 \cdot \lambda / 2$ long. This length provides the lowest emittance growth. 
made of high purity niobium. The cavity, equipped with two HOM couplers, input coupler and pickup probe will be assembled for operation in a dedicated cryostat. The gun is designed to be implemented in the split injector. A solenoid, installed directly after the cavity, will be used for emittance growth compensation. Tables 1 and 2 and Figure 3 show RF-parameters and the Higher Order Mode (HOM) suppression $\left(Q_{\text {ext }}\right)$ for the present design. The damping of HOMs in a SRF-gun is crucial for the beam quality, which can be diluted by the interaction between non-relativistic electrons (in the first 0.6-cell) with deflecting dipole modes. The operating electric field on the lead cathode $E_{\text {peak }}$ will not exceed $60 \mathrm{MV} / \mathrm{m}$. At this field, the emitting spot will be exposed to $B=4 \mathrm{mT}$, which is much smaller than the lead critical magnetic flux $B_{c}=70 \mathrm{mT}$.

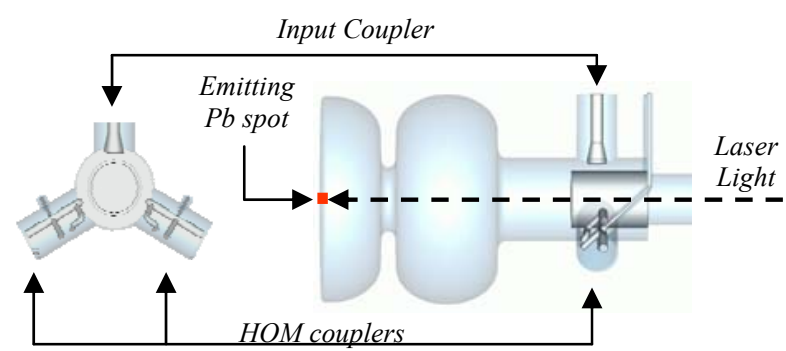

Figure 2: 1.6-cell SRF-gun cavity with 2 HOM couplers and the input coupler.

Table 1: RF-parameters of the 1.6-cell SRF gun

\begin{tabular}{|l|c|c|}
\hline \multicolumn{1}{|c|}{ Parameter } & Unit & Value \\
\hline$\pi$-mode frequency & {$[\mathrm{MHz}]$} & 1300 \\
\hline 0-mode frequency & {$[\mathrm{MHz}]$} & 1286.5 \\
\hline Cell-to-cell coupling & - & 0.015 \\
\hline Active length $1.6 \cdot \lambda / 2$ & {$[\mathrm{~m}]$} & 0.185 \\
\hline Nominal $E_{\text {cath }}$ at cathode & {$[\mathrm{MV} / \mathrm{m}]$} & 60 \\
\hline Energy stored at nominal $E_{\text {cath }}$ & {$[\mathrm{J}]$} & 20 \\
\hline Nominal beam energy & {$[\mathrm{MeV}]$} & 6 \\
\hline
\end{tabular}

Table 2: HOMs of 1.6-cells

\begin{tabular}{|l|c|l|}
\hline \multicolumn{1}{|c|}{ Mode } & $f[\mathrm{MHz}]$ & \multicolumn{1}{c|}{$(R / Q)$} \\
\hline Dipole: TE111-1a & 1641.8 & $1.85\left[\Omega / \mathrm{cm}^{2}\right]$ \\
\hline Dipole: TE111-1b & 1644.9 & $1.30\left[\Omega / \mathrm{cm}^{2}\right]$ \\
\hline Dipole: TM110-1a & 1883.5 & $10.1\left[\Omega / \mathrm{cm}^{2}\right]$ \\
\hline Dipole: TM110-1b & 1884.0 & $9.99\left[\Omega / \mathrm{cm}^{2}\right]$ \\
\hline Dipole: TM110-2a & 1957.0 & $3.90\left[\Omega / \mathrm{cm}^{2}\right]$ \\
\hline Dipole: TM110-2b & 1957.1 & $3.85\left[\Omega / \mathrm{cm}^{2}\right]$ \\
\hline Monopole: TM011 & 2176.5 & $43.2[\Omega]$ \\
\hline
\end{tabular}

Two types of half-cell resonators have been built to measure the $Q E$ of lead at $2 \mathrm{~K}$ and to test the $\mathrm{RF}$ performance of $\mathrm{Nb}-\mathrm{Pb}$ cavities. Both types are shown in Fig. 4. The left one was built at TJNAF. It has an opening in the center of the back wall, which is vacuum sealed with a niobium plug and an indium gasket. The advantage of this cavity type is that plugs can be easily coated with emitting materials to test various deposition methods and various superconductors for photoemission.

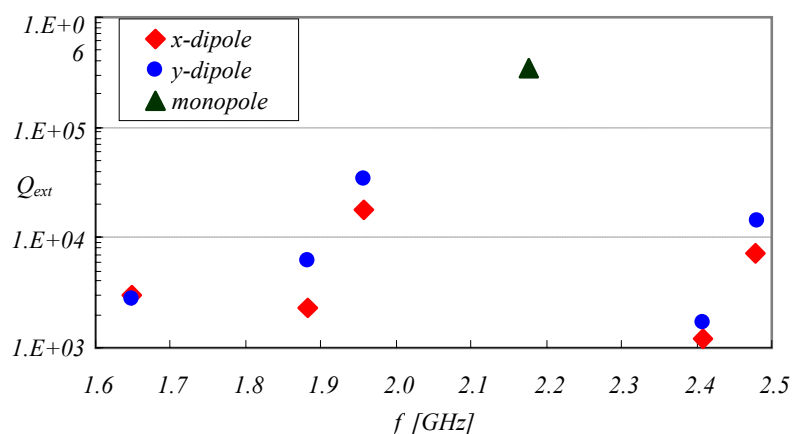

Figure 3: 3D modelling of the HOM suppression for the 1.6-cell SRF-gun.

It can be an alternative solution to the second cavity type, built at DESY (Fig. 4, right), in which, the technically difficult coating is done directly on the back wall. An additional difficulty in this version is that the emitting spot must withstand cleaning procedures applied to the cavity. Two of the cleaning steps could degrade $Q E$ : chemical treatment and high pressure water rinsing. Both procedures are essential for good performance of $\mathrm{sc}$ cavities. On the other hand, two features make this version very attractive: the smooth back wall does not enhance locally the electric field near the cathode and there is no RF electric contact needed in the high field region, which may reduce the intrinsic $Q_{o}$ of the cavity. The plug cavity and the first DESY cavity were tested several times without and with lead coating. The baseline test without the coating, of the second DESY cavity fabricated in 2006, was recently carried out at TJNAF.

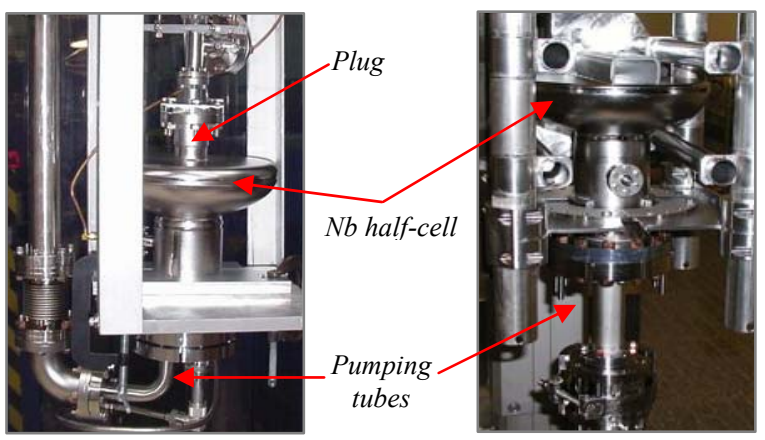

Figure 4: Test half-cell cavities built at TJNAF (left) and at DESY (right) assembled on inserts for the RF-cold test.

\section{RF-PERFORMANCE TEST}

\section{Cold test without coating}

Figure 5 displays the best results for the half-cell cavities without lead coating on the back wall and on the plug. The tests showed that chemical treatment and high pressure water rinsing are challenging for the half-cells and need to be improved in the future. We observed, testing DESY type cavities, few multipacting levels between 30 and $40 \mathrm{MV} / \mathrm{m}$, which could be processed for cavities without lead. It took some time to overcome these levels by RF-processing. In the plug cavity, we observed heating of the first plug version, which was due to insufficient cooling by LHe. The cooling was improved for the tests shown. 


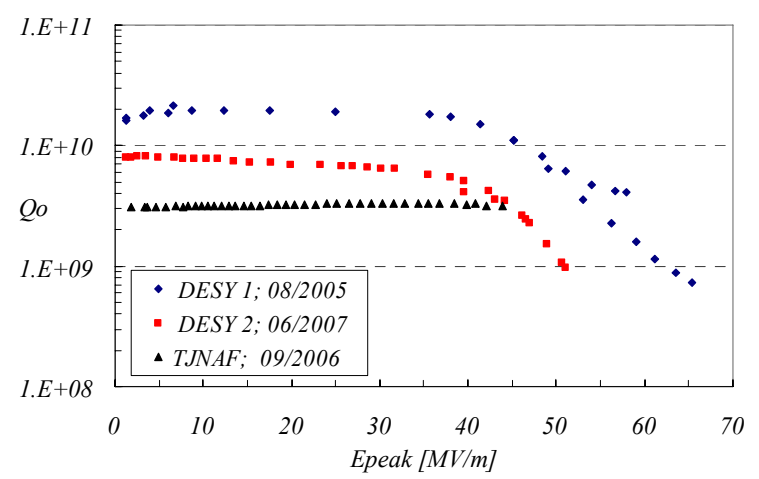

Figure 5: Cold (2K) RF-test results without lead coating.

\section{Cold test with lead coating}

The emitting $4 \mathrm{~mm}$ diameter lead spot at the center of the back wall of the DESY cavity was deposited by the arc-discharge method at A. Soltan INS. The $7 \mathrm{~mm}$ diameter plug of the TJNAF cavity was electroplated with lead at Stony Brook University. As mentioned before, the final cleaning of the cavities is challenging, especially for the DESY type when coated. That cavity reached 40 $\mathrm{MV} / \mathrm{m}$ peak field (Fig. 6), but a lot of processing events and heavy radiation was observed during the cold test. The second DESY cavity will be coated and tested soon. The TJNAF cavity is somewhat easier to clean because of the absence of the coated plug during surface treatment. It demonstrated almost the same performance as without lead, which confirms our expectation that the emitting spot located on axis should not limit the performance.

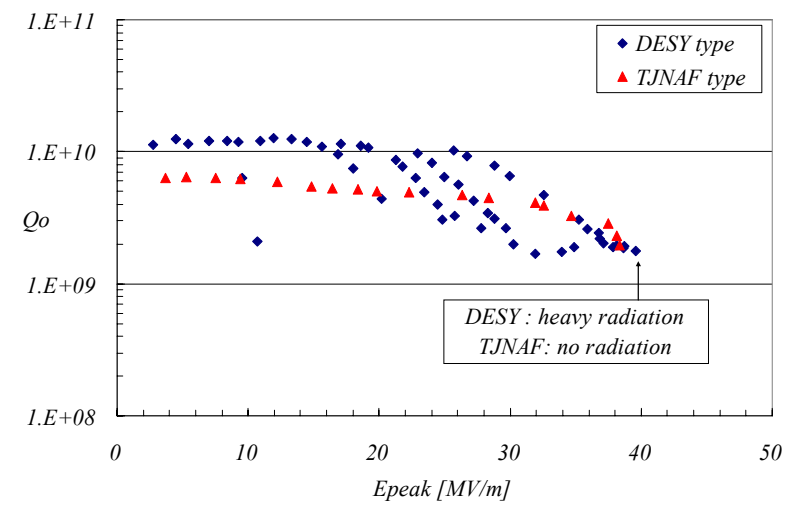

Figure 6: Cold (2K) RF-test results with lead coating.

\section{Irradiation with laser}

One of our concerns from the very beginning of this project was the recombination time of broken Cooper pairs in the emitting spot by the irradiating laser. The theoretical investigation presented in [1] led to the conclusion that the recombination time strongly depends on temperature. Fortunately, the time is shorter for higher temperature. The $248 \mathrm{~nm}$ excimer laser used for the cold tests generated $5.3 \mathrm{~ns}$ long pulses (FWHM) at up to 250 $\mathrm{Hz}$ repetition frequency. The maximum energy per pulse was $5.5 \mathrm{~mJ}$. Half of it could be transferred to the cavity for the $Q E$ and relaxation time experiments. The maximum available peak power of $0.518 \mathrm{MW}$ at the cathode was two times higher than the power needed for the nominal operation of the gun, when $1 \mathrm{nC}$ bunches are generated within 20 ps. The preliminary relaxation time experiment was performed with the TJNAF cavity, operating at $1.42 \mathrm{GHz}$. For that experiment the cavity had to be attached to a straight vacuum tube oriented vertically upward, to enable direct irradiation with the laser light via the sapphire window installed at the topplate of the vertical cryostat. The $3 \mathrm{~m}$ long vacuum tube contaminated the cavity with particulates and degraded its performance. At $3 \mathrm{MV} / \mathrm{m} Q_{o}$ was only $2.25 \cdot 10^{9}$, almost 3 times lower than $Q_{o}$ measured at this gradient in the RFperformance test. When the $\mathrm{Nb}$ wall was irradiated with the maximum available laser power, $Q_{o}$ dropped to $\sim 1.6 \cdot 10^{9}$, but the cavity did not quench and behaved still very stable. The additional dissipation, due to the locally broken Cooper pairs in the irradiated area, was $5.2 \mathrm{~mW}$. The surface resistance, $r_{s}$, of the irradiated area increased during the laser pulse by a factor of 630 . The ratio of $r_{s}$ after and before the irradiation indicates that the concentration of quasi-particles rose to their equilibrium concentration at $8 \mathrm{~K}$. According to the theoretical model, their relaxation time is shorter than $100 \mathrm{ps}$ at this temperature.

\section{ACKNOWLEDGMENT}

We would like to express our gratitude to colleagues at DESY, BNL, INS and TJNAF from whom we received technical support.

\section{REFERENCES}

[1] J. Sekutowicz et al., "Nb-Pb Superconducting RFgun", TESLA-FEL Report 2005-09, DESY, 2005.

[2] M. Ferrario, "Low emittance photo-injectors", NIM A472, (2001), 303-308.

[3] J. Sekutowicz, "Superconducting RF Photoinjectors; an Overview", Proceed. ICFA Workshop on The Physics and Applications of High Brightness Electron Beams, Erice, Italy, 9-14 October, 2005; http://www.physics.ucla.edu/PAHBEB2005/talks/10 oct 2005/index.htm

[4] A. Michalke et al., "First Operation of the High Quantum Efficiency Photocathodes Inside Superconducting Cavities", Proceed. EPAC92, Berlin, Germany, 1992.

[5] D. Janssen, P. vom Stein, "The Development of a Superconducting RF Gun: Status of the Drossel Collaboration", APS Proceed. PAC97, Vancouver, Canada, 1997.

[6] T. Srinivasan-Rao et al., "Design, Construction and Status of All Niobium Superconducting Photoinjector at BNL", APS Proceed. PAC03, Portland,USA, 2003.

[7] J. Sekutowicz et al., "Nb-Pb Superconducting RFGun”, TESLA-FEL Rep.-2005-09, DESY, 2005.

[8] J. Smedley et al., "Photoemission Properties of Lead", Proceed. EPAC2004, Lucerne, Switzerland, 2004.

[9] J. Smedley et al., "Photoemission Tests of a $\mathrm{Pb} / \mathrm{Nb}$ Superconducting Photoinjector", these Proceedings 